\title{
VAdriaC Regimen
}

National Cancer Institute

\section{Source}

National Cancer Institute. VAdriaC Regimen. NCI Thesaurus. Code C67231.

A regimen consisting of vincristine, doxorubicin and cyclophosphamide, used for the treatment of localized childhood Ewing's sarcoma. 